\title{
Thermally Induced Density Perturbations in the Inflation Era
}

\author{
Arjun Berera* and Li-Zhi Fang \\ Department of Physics, University of Arizona, Tucson, 85721
}

(November 5, 2018)

\begin{abstract}
The possibility of thermally induced initial density perturbations in inflationary cosmology is examined. The fluctuation dynamics of a scalar field plus thermal bath system during slow roll is described by a Langevin-like equation. Fluctuation-dissipation arguments show that for a wide parameter range within the standard inflation model, the thermal fluctuations of the scalar field can dominate its quantum fluctuations. The initial amplitude of density perturbations is found to lie in a range which is consistent with the recent observations of cosmic temperature fluctuations.
\end{abstract}

PACS numbers: 98.80.Cq, 05.40.+j

The standard scenario for structure formation in the universe is based on inflationary cosmology. According to this model, quantum fluctuations of the scalar field during the expansion era were the perturbing seeds in an initial, globally smooth universe. From this, largescale structures then arose [1]. This model predicts that the initial density perturbations should be Gaussian, and have a power-law spectrum with index $n \sim 1$. Based on this model for the initial perturbations plus assumptions about dark matter, the formation of galaxies, clusters of galaxies and other objects have been extensively studied [2]. Subsequent studies have shown that this model is consistent with observation, thus not theoretically implausible. Recent detection of temperature anisotropies in cosmic background radiation (CBR) by the Differential Microwave Radiometer (DMR) on the Cosmic Background Explorer's $(C O B E)$ satellite has given the first opportunity to directly probe the initial density perturbation. These results fit the scaling spectrum given by the inflation model [3]. Thus it is one source of support for the inflation model in describing the initial density perturbations.

However, the COBE-DMR results do raise questions about the amplitude of the initial perturbations. Such questions are not new, only further perpetuated by the $C O B E$ results. Before $C O B E$ it was already possible to determine the amplitude by fitting (or normalizing) the evolved perturbations with the observed clustering of galaxies on scales of say $8 \mathrm{~h}^{-1} \mathrm{Mpc}$, where $h$ is the Hubble constant in unit of $100 \mathrm{~km} \mathrm{~s}^{-1} \mathrm{Mpc}^{-1}$. This method suffers from three uncertainties arising from: 1) the evolution of the perturbations; 2) the assumptions of dark matter, and 3) the bias factor. On the other hand, the temperature fluctuations of CBR on scales of superhorizons size at the decoupling time, i.e. larger than about $2^{\circ}$, directly provide information about the initial density perturbation, independent of the above three factors. The $C O B E$ result of the CBR quadrupole amplitude has already been used to normalize the amplitude of the cosmic density perturbations. Therefore, it is now a question of observational importance to explain the so found amplitude of the initial perturbations.

The origin of the amplitude's magnitude is also rele- 
vant because the "standard" scenario cannot explain it naturally. It has been known since developing the inflation model, that the amplitude of the initial density perturbations given by quantum fluctuations of the inflationary scalar field is, at least for the "standard" model, of order 1 , which is about $10^{4}$ times larger than the required value [4]. This is sometimes called the fluctuation problem. One can relax this conflict by sophisticated designs for the inflationary potential. However, such particle physics designs counter the naturality philosophy of inflation. Moreover, in these models the amplitude is completely determined by unknown parameter(s) of the so designed potential. This gives almost no constraint to the possible or reasonable range for the amplitude. As such, it cannot predict what the initial value of the perturbation amplitude should be.

This situation motivates a search other possible mechanisms, which do not depend on such sophisticated design. In this paper we show how thermal fluctuations during inflation may actually play the dominant role in producing the initial perturbations. It is conventionally believed that the components of particle-like matter, either relativistic or non-relativistic, are totally negligible during inflation. This is certainly true if we only consider the energy density, because inflation is by definition the epoch when the vacuum energy of the scalar field was the dominant component in the universe. However, this does not imply that the initial perturbations must mainly arise from quantum fluctuation of the scalar field. All particle-like matter which existed before inflation would have been dispersed by inflation. Yet, particle-like matter will not completely vanish if one considers the processes of the scalar field dissipating into a thermal bath via its interaction with other fields.

The existence of a thermal component during inflation may not be exceptional and perhaps even inevitable. Roughly one can see this as follows. In order to maintain the $\phi$ field close to its minimum at the onset of the inflation phase transition, thermal forces will generically be an important contributing source. Therefore, at least in the starting period of the phase transition, there is thermal contact between $\phi$ and all other fields with which it interacts. During the slow roll period of inflation, the kinetic and potential energy of the $\phi$ field is fairly constant, so that the interaction between the $\phi$ field with the other fields remains about the same as at the beginning. As such, there is no compelling reason to believe that the thermal component vanishes during inflation.

We will show that, for a wide range of the parameters characterizing inflation, there can be a thermal component in the universe. With account for such a component, we find that the density perturbations can originate mainly from thermal fluctuations. Accepting this mechanism, the fluctuation problem would be automatically avoided. In addition the allowable range for the amplitude would be consistent with the observed results.

Let us consider the standard model of inflation, given by a scalar field $\phi$ with Lagrangian density 


$$
L=\frac{1}{2} \partial^{\mu} \phi \partial_{\mu} \phi-V(\phi)+L_{i n t}
$$

where $V(\phi)$ is the effective potential, and $L_{\text {int }}$ describes the interaction of $\phi$ with all other fields. The classical equation of motion for $\phi$ in a de-Sitter universe is

$$
\ddot{\phi}+3 H \dot{\phi}+\Gamma_{\phi} \dot{\phi}-e^{-2 H t} \nabla^{2} \phi+V^{\prime}(\phi)=0 \text {. }
$$

The friction term $\Gamma_{\phi} \dot{\phi}$ phenomenologically describes the decay of the $\phi$ field via the interaction Lagrangian $L_{i n t}$. In principle, the friction term $\Gamma_{\phi} \dot{\phi}$ may not be reasonable for describing the energy transfer from $\phi$-field particle production during far out of equilibrium conditions. However, it is a proper approximation for describing the energy dissipated by the $\phi$ field into a thermalized radiation bath. It should also be noted that $\Gamma_{\phi}$ could be a function of $\phi$. Due to the lack of a detailed model for the decay of $\phi$, we assume that $\Gamma_{\phi}$ is a constant independent of $\phi$. As we will show, the thermal fluctuations of $\phi$ do not depend significantly on the details of $\Gamma_{\phi}$.

In the standard treatment of the inflation model, one assumes that the inflation era is divided into two regimes: 1) slow roll and 2) reheating. In the former all interactions between the inflationary scalar field and other fields are typically neglected. These interactions have been considered only in the latter, in order to supply the mechanism to reheat the universe. This is equivalent to assuming that the friction term is only important in the reheating regime, but negligible during the slow-roll (inflation) regime, i.e. $3 H \gg \Gamma_{\phi}$. In this case, the slow-roll evolution in a spatially homogeneous universe is given by

$$
\dot{\phi} \simeq-\frac{V^{\prime}(\phi)}{3 H},
$$

where $H^{2}=(8 \pi G / 3) \rho_{\phi} \sim(8 \pi / 3) M^{4} / m_{p l}^{2}, \rho_{\phi}=\dot{\phi}^{2} / 2+$ $V(\phi)$ is energy of the $\phi$ field, $m_{p l}=(1 / G)^{1 / 2}$ is the Planck mass, and $V(\phi) \sim V(0)=M^{4}$. Eq.(3) is valid when the potential $V(\phi)$ satisfies the following well known conditions for the inflationary potential [1],

$$
\begin{gathered}
\left|V^{\prime \prime}(\phi)\right| \ll 24 \pi V(\phi) / m_{p l}^{2}, \\
V^{\prime 2}(\phi) m_{p l}^{2} \ll 48 \pi V^{2}(\phi) .
\end{gathered}
$$

Our first observation is that the condition $\Gamma_{\phi} \ll 3 H$ is not necessary for a slow roll solution. The coupling of the inflationary scalar field with other fields can co-exist with the roll down solution. Consider the case when $\Gamma_{\phi}$ is comparable to $H$. This implies that its decay products will equilibrate quickly to some temperature $T_{r}$. For explicitness in the treatment below, let us make the reasonable assumption that the decay products of the $\phi$-field are relativistic matter. The additional equation needed to describe this relativistic component from the first law of thermodynamics is

$$
\dot{\rho}_{r}+4 H \rho_{r}=\Gamma_{\phi} \dot{\phi}^{2}
$$


where $\rho_{r}$ is the energy density of the thermal component. With account for this component, the slow roll equation (3) should be replaced by the set

$$
\begin{gathered}
\dot{\phi} \simeq-\frac{V^{\prime}(\phi)}{3 H+\Gamma_{\phi}} \\
\dot{\rho}_{r} \simeq 0 .
\end{gathered}
$$

and

$$
H^{2}=\frac{8 \pi G}{3}\left(\rho_{\phi}+\rho_{r}\right) .
$$

Strictly speaking, when there is a thermal component in the universe, one should use a finite temperature effective potential to replace the zero-temperature potential $V(\phi)$. As we will show below, this replacement will not be important for our purpose.

Solution (7) implies that independent of the initial conditions for the thermal component, it will reach a steady state regime during inflation, i. e. the depletion of the radiation due to expansion will be balanced by its production due to friction. From eqs.(5) - (7), the constant energy density of the thermal component is found to be

$$
\rho_{r} \simeq \frac{\Gamma_{\phi}}{4 H} \dot{\phi}^{2}
$$

In the inflation epoch the kinetic energy of the $\phi$ field, $\dot{\phi}^{2} / 2$, is much less than its vacuum energy $\rho_{\phi} \sim V(\phi)$. Thus we have $\rho_{r} \ll \rho_{\phi}$ if

$$
\Gamma_{\phi} \leq \alpha 4 H
$$

where $\alpha>1$ is a model dependent arbitrariness. As such, in terms of energy density, the considered system during inflation is still dominated by the vacuum energy of the $\phi$ field, with in particular the thermal component being negligible. Thus other aspects of the the inflationary scenario will remain the same as in the standard model.

However, in terms of the system's temperature, the presence of a thermal component is not necessarily negligible. The temperature, $T_{r}$, of the thermal component is given by

$$
T_{r} \simeq \rho_{r}^{1 / 4} \simeq\left(m_{p l} W \Gamma_{\phi}\right)^{1 / 4} M^{1 / 2},
$$

where $W=\dot{\phi}^{2} / 2 V(\phi)$ is the ratio of the kinetic and potential energy of the $\phi$ field. From eqs. (9), (10) and (11), it is easy to show that the temperature of the thermal component can be greater than the Hawking temperature, i.e.

$$
T_{r}>H
$$

if

$$
\Gamma_{\phi}>\left(\frac{M}{m_{p l}}\right)^{5} \frac{M}{W} .
$$


All conditions (4), (10) and (13) can be simultaneously satisfied if

$$
V^{3 / 2}(\phi) m_{p l}^{-3} \ll V^{\prime}(\phi) \ll m_{p l}^{-1} V(\phi)
$$

Since $\left(M / m_{p l}\right)^{2} \ll 1$, this condition can be fulfilled. In fact, there is big room in the parameter space of the potential, in which the inflationary expansion of the universe will still be dominated by the scalar field, but the temperature of the system will be determined by the thermal matter $\rho_{r}$. On the other hand, because $W \ll 1$ during the slow-roll regime, eq.(11) implies that $T_{r} \ll M$. Using $\lambda \phi^{4}$ as a generic inflationary potential, the leading temperature effect is known to be $\lambda T_{r}^{2}$ [1]. Recalling that to have inflation requires $\lambda \ll\left(M / m_{p l}\right)^{2}$ means $\lambda T_{r}^{2} \ll M^{4} / m_{p l}^{2} \sim H^{2}$. Therefore, the influence of the finite temperature effective potential is insignificant as stated earlier.

The quantum mechanical fluctuations of the $\phi$ field during inflation are determined by the Hawking temperature $H$. Therefore, one can expect that eq.(12) is the condition under which thermal fluctuations will compete with quantum fluctuation. We will justify this point in the following.

To calculate the fluctuations, the $\phi$ field should be treated as a stochastic field. Therefore, eq.(6) should be interpreted as the ensemble averaged equation of motion. The essence of eq.(6) can easily be seen if it is rewritten as

$$
\frac{d \phi}{d t}=-\frac{1}{3 H+\Gamma_{\phi}} \frac{d F[\phi]}{d \phi},
$$

where $F[\phi]=V(\phi)$. Eq.(15) is, in fact, a equation for the rate of change of the order parameter $\phi$ of a homogeneous system with free energy $F[\phi]$. It describes the approach to equilibrium for the system. More generally in a study of fluctuations, one should not use the approximation of a spatially homogeneous universe, so that the spatial gradient term $\exp (-2 H t) \nabla^{2} \phi$ in eq.(2) should not be ignored. The equation (15) for the rate of change is then modified to

$$
\frac{d \phi(\mathbf{x}, t)}{d t}=-\frac{1}{3 H+\Gamma_{\phi}} \frac{\delta F[\phi(\mathbf{x}, t)]}{\delta \phi}
$$

where the free energy is given by

$$
F[\phi]=\int d^{3} \mathbf{x}\left[\frac{1}{2}\left(e^{-H t} \nabla \phi\right)^{2}+V(\phi)\right] .
$$

Our present purpose is limited to comparing the amplitudes of thermal and quantum fluctuations. For this, it is enough only to calculate the $\phi$-field fluctuations for the mode of wavelength equal to the horizon $H^{-1}$ during inflation. As such the contribution from the spatial gradient term for only this mode is important in calculating the correlation function of the scalar field.

It is known that rate equations like (17) cannot correctly describe the approach to equilibrium during a 
phase transition without also a noise term [5]. For instance, eq.(17) will only cause the order parameter to evolve towards local minima but not necessarily the global minimum. To ensure that the system approaches the global minimum, we must remember that actually the order parameter dynamics is not purely relaxational, but may exhibit fluctuations, arising from the microscopic degrees of freedom. These fluctuations can be modeled by introducing a noise term $\eta$ into eq.(17) as

$$
\frac{d \phi}{d t}=-\frac{1}{3 H+\Gamma_{\phi}} \frac{\delta F}{\delta \phi}+\eta(t) .
$$

This is the Langevin equation for a system with one degree of freedom, in similar analogy to say a Brownian particle. A similar type of equation has been examined in [6]. However, their purpose was to statistically treat the quantum fluctuations of the scalar field, whereas ours is to treat external thermal forces.

We had shown in [7] that during the eras of dissipations, the dynamics of structure formation in the universe should be described by a KPZ-equation [8], which describes systems governed by non-linear effects plus stochastic fluctuations. Eq.(18) is a realization of this hypothesis, within the context of scalar field dynamics in the standard inflationary model. Although it is in all generality non-linear, in this paper we will not study the complete spectrum of the density perturbations, but only the amplitude of fluctuations with wavelength $\sim H^{-1}$. For this we will not concentrate on the non-linear effects in eq. (18).

The stochastic force $\eta$ in eq.(18) can be found from the fluctuation-dissipation theorem [9]. If the temperature of the thermal bath is $T_{r}$, the expectation values of $\eta$ is given by

$$
\langle\eta(t)\rangle_{\eta}=0
$$

and

$$
\left\langle\eta(t) \eta\left(t^{\prime}\right)\right\rangle_{\eta}=D \delta\left(t-t^{\prime}\right) .
$$

The notation $\langle\ldots\rangle_{\eta}$ denotes averaging of $\eta$ with respect to a Gaussian distribution. The variance $D$ is given by

$$
D=2 \frac{1}{U} \frac{T_{r}}{3 H+\Gamma_{\phi}}
$$

where $U=(4 \pi / 3) H^{-3}$ is the volume with Hubble radius $H^{-1}$.

The fluctuations $\delta \phi$ of the $\phi$ field can be found from linearizing eq.(18). If we only consider the fluctuations $\delta \phi$ crossing outside the horizon, i.e. with wavelength $\sim$ $H^{-1}$. The equation of $\delta \phi$ is

$$
\frac{d \delta \phi}{d t}=-\frac{H^{2}+V^{\prime \prime}(\phi)}{3 H+\Gamma_{\phi}} \delta \phi+\eta .
$$

According to condition (4), the term $V^{\prime \prime}(\phi)$ on the right hand side of eq.(22) can be neglected. From eq. (22), one finds for the correlation function of the fluctuations 


$$
\left\langle\delta \phi(t) \delta \phi\left(t^{\prime}\right)\right\rangle_{\eta} \simeq D \frac{3 H+\Gamma_{\phi}}{2 H^{2}} e^{-\left(t-t^{\prime}\right) H^{2} /\left(3 H+\Gamma_{\phi}\right)}, \quad t>t^{\prime},
$$

so that

$$
\left\langle(\delta \phi)^{2}\right\rangle_{\eta} \sim \frac{3}{4 \pi} H T_{r}
$$

This is our central result. Notice that it is independent of $\Gamma_{\phi}$. This is expected since it is simply the variance of the $\phi$ field when coupled to a thermal bath, as implicit to the fluctuation-dissipation theorem. Nevertheless, the importance of a sufficiently large decay term, as emphasized earlier, is to ensure appropriate dynamical conditions for rapid thermalization of the radiation bath on the scale of the expansion rate $H$. From eq.(24) one can conclude that the thermal fluctuations of the scalar field will be greater than its quantum fluctuations, $\left\langle(\delta \phi)^{2}\right\rangle_{Q M} \sim H^{2} / 2 \pi$, when condition (12) holds.

Since the kinematics and dynamics of inflation are the same here as in the standard model, the initial perturbations will still have a power-law spectrum with index $n \sim 1$. Also the amplitude $\delta \rho / \rho$, when it crosses back inside the horizon, can be calculated by the gauge invariant amplitude $\zeta=\delta \rho_{\phi} /(\rho+p)$ during the time of inflation. For quantum fluctuations it is known that $\zeta$ is of order 1. This follows simply because for a field in its ground state, the mean quantum fluctuation of its energy density is of the same order as its mean kinetic energy density. However, for thermal fluctuations, the mean kinetic energy density will be greater than its fluctuation, so one can except that the quantity $\zeta$ should be much less than 1.

The energy fluctuations caused by $\delta \phi$ is $\delta \rho=\delta \phi V^{\prime}(\phi)$, and $\rho+p=\rho_{\phi}+p_{\phi}+\rho_{r}+p_{r}=\dot{\phi}^{2}+(4 / 3) \rho_{r}$. Therefore from eqs. $(6),(9),(11)$ and (24), we have

$$
\frac{\delta \rho}{\rho} \simeq \frac{\delta \phi V^{\prime}(\phi)}{\dot{\phi}^{2}+(4 / 3) \rho_{r}} \simeq \frac{3}{4}\left(\frac{3 \Gamma_{\phi}}{\pi H}\right)^{1 / 2}\left(\frac{H}{T_{r}}\right)^{3 / 2}
$$

Because $T_{r}<M$, eq.(25) shows that the amplitude of the initial perturbations, $\delta \rho / \rho$, should be in the following range,

$$
\left(\frac{M}{m_{p l}}\right)^{3 / 2} \ll \frac{\delta \rho}{\rho}\left(\frac{H}{\Gamma_{\phi}}\right)^{1 / 2} \ll 1 .
$$

Therefore, the amplitude of the initial perturbations is mainly limited by the ratio $M / m_{p l}$, i.e. the energy scale of inflation. If we take $M \sim 10^{15} \mathrm{Gev}$, the possible range for the amplitude $\delta \rho / \rho$ should be in about the middle of the range $\left(10^{-6}-1\right)\left(\Gamma_{\phi} / H\right)^{1 / 2}$. This result is consistent with the observed amplitude $\delta \rho / \rho \sim 10^{-4}$.

As an additional outcome of this treatment, eq. (26) places an upper limit to the energy scale of inflation of $M<m_{p l}(\delta \rho / \rho)^{2 / 3} \sim m_{p l} \cdot 10^{-3}$, above which thermally induced fluctuations would be inconsistent with the observed density perturbations. Therefore, one can also 
conclude that for thermally caused initial perturbations, inflation should not occur earlier than about $m_{p l} / 10^{2}$.

In addition to the amplitude fluctuation for the scalar mode, which was treated in this paper, there is also an amplitude fluctuation for the tensor mode 10]. Since this involves weakly interacting gravitons, a thermal mechanism for inducing these fluctuations seems less likely to the standard treatment which considers quantum fluctuations.

Financial support was provided by the NSF INT9301805 grant and the U. S. Department of Energy, Division of High Energy and Nuclear Physics.

* Present address: Department of Physics, the Pennsylvania State University, 104 Davey Laboratory, University Park, PA 16802-6300

[1] See for example, E.W.Kolb and M.S. Turner, The Early Universe, (Addison-Wesley, New York, 1990).

[2] P.J.E.Peebles, Principles of Physical Cosmology, (Princeton, 1993).

[3] G. Smoot et al., Astrophys. J. 396, L1 (1992); C.L. Bennett, et al., Astrophys. J. (1994) in press.

[4] J.M. Bardeen, P.J. Steinhardt, \& M.S. Turner, Phys. Rev. D28, 679 (1983); A. Linde, Phys. Lett. 129B, 177 (1983); R. Brandenberger \& R. Kahn, Phys. Rev. D28, 2172 (1984); G. Mazenko, W. Unruh \& R. Wald, Phys. Rev. D9, 3357 (1985).

[5] See for example, N. Goldenfeld, Lectures on Phase Transitions and the Renormalization Group, (Addison Wesley, 1992).

[6] A.A. Starobinsky, in Fundamental Interactions (MGPI Press, Moscow, 1984), p. 55; A.S. Goncharov and A.D. Linde, Sov. J. Part. Nucl. 17, 369 (1986); S. J. Rey, Nucl. Phys. B284, 706 (1987); D.S. Salopek \& J.R. Bond, Phys. Rev. 43D, 1005 (1991) and references therein.

[7] A. Berera and L.Z. Fang, Phys. Rev. Lett. 72, 458 (1994).

[8] M. Kardar, G. Parisi, and Y. C. Zhang, Phys. Rev. Lett. 56, 342 (1986).

[9] P.C. Hohenberg \& B.I. Halperin, Rev. Mod. Phys. 49, 435 (1977).

[10] A. A. Starobinsky, JEPT Lett. 30, 682 (1979). 